\title{
An Interpretation of Woodward's Ambiguity Function and Its Generalization
}

\author{
John E Gray \\ Code Q-23 \\ Electromagnetic and Sensor Systems Department \\ Naval Surface Warfare Center Dahlgren \\ 18444 Frontage Road Suite 328 \\ Dahlgren, VA 22448-5161, USA \\ Email: DLGR-NSWC-Q23@navy.mil
}

\begin{abstract}
We suggest a new interpretation of Woodward's ambiguity function as the expected value of an operator. The operator represents the physics of the interaction of the waveform with the object. This approach provides a new approach to understanding the return signal at the receiver and can reveal more detailed understanding of the underlying interactions within the return signal that are not usually brought out by standard signal processing techniques.
\end{abstract}

\section{CONTEnTs}

\section{Introduction}

\section{Operator Approach}

\section{Examples}

III-A Single Dimensional Signals . . . . . . . 4

III-B Cross Operator Ambiguity Functions . .

\section{Conclusions}

\section{References}

\section{INTRODUCTION}

Woodward introduced the ambiguity function in the seminal book "Probability and Information Theory with Applications to Radar"[1] as the means to solve the measurement problem of radar. The measurement problem of radar is to design a radar waveform to be broadcast by a radar or sonar, so as to maximize the receiver response to the signal which has interacted with an object. The means to accomplish this was drawn from work by North[2] during WW II who invented the "matched filter". A matched filter is obtained by correlating a known signal template with an unknown signal to detect the presence or absence of the template in the unknown signal. This is exactly equivalent to convolving the unknown signal with the complex conjugate of the time-reversed version of the known signal template which; this is termed cross-correlation. Therefore, the matched filter is the optimal linear filter for maximizing the signal to noise ratio (SNR) in the presence of additive noise as has been shown in many texts[3],[4],[5]. In radar, a known signal is sent out and the reflected signal from the object (which is a function of the distance to the object, the relative speed of the object and the broadcast frequency of the radar), can be examined at the radar receiver for the common elements of the out-going signal in the return signal, which, when optimized is a multi-dimensional matched filter or ambiguity function. Formally, the form of the return signal for a narrow band signal $s(t)$ is $s(t-\tau)$ where $\tau$ is the delay between the broadcast of the signal and the return time that the signal is detected at the receiver. For the broadband form of the signal, the form of the return signal is $s(\alpha(t-\tau))$ where $\tau$ is the delay and

$$
\alpha=\frac{c-v}{c+v}=\frac{1-\beta}{1+\beta}
$$

where $c$ is the speed of light and $v$ is the radial velocity of the object.

Thus, there are two common forms of the ambiguity function: the narrowband ambiguity function, $\chi_{N},\left(s^{*}(t)\right.$ means complex conjugate of the broadcast signal)

$$
\chi_{N}(\omega, \tau)=\int_{-\infty}^{\infty} e^{-i t \omega} s^{*}(t) s(t-\tau) d t
$$

and the wideband ambiguity function, $\chi_{W B}$,

$$
\chi_{W B}(\omega, \tau)=\sqrt{\alpha} \int_{-\infty}^{\infty} s^{*}(t) s(\alpha(t-\tau)) e^{-i t \omega} d t
$$

For a discussion about how to design radar signals so that their ambiguity functions have specific desirable properties useful for various kinds of radars, Leavon is a current up to date resource[7]. There is another way to think about at the ambiguity function that is different than the way Woodward presented it. This approach suggests the radar measurement problem, can be cast in a more abstract setting, that treats interaction between the waveform and the target can be treated as an operator acting on the waveform. This approach can be termed the operator approach, it can be viewed as an abstraction of the quantum mechanical formalism to a classical setting. This approach has been championed by Cohen[8] as a way for recasting problems in signal processing. 


\section{Operator APPROACH}

The notation for the inner product of two signals, $r(t)$ and $s(t)$ that is used throughout the paper is

$$
\langle r(t), s(t)\rangle=\int_{-\infty}^{\infty} r^{*}(t) s(t) d t,
$$

while the Fourier transform, $\mathcal{F}$, of a signal $s(t)$ is[6]

$$
S(\omega)=\mathcal{F} s(t)=\int_{-\infty}^{\infty} e^{-i t \omega} s(t) d t=\left\langle e^{i t \omega}, s(t)\right\rangle,
$$

while the inverse Fourier transform, $\mathcal{F}^{-1}$, is

$$
s(t)=\mathcal{F}^{-1} S(\omega)=\frac{1}{2 \pi} \int_{-\infty}^{\infty} e^{i t \omega} S(\omega) d \omega=\left\langle e^{-i t \omega}, S(\omega)\right\rangle .
$$

A function of time which is translated by amount, $\tau$, can be written as (using the Taylor expansion of function $D=\frac{d}{d t}$ )

$$
s(t+\tau)=e^{\tau D} s(t)=e^{i\left(-i \tau \frac{d}{d t}\right)} s(t)=e^{i \tau \mathcal{W}} s(t)
$$

The form of $\chi_{N}(\omega, \tau)$, it can be recast as

$$
\begin{aligned}
\chi_{N}(\omega, \tau) & =\int_{-\infty}^{\infty} e^{-i t \omega} s^{*}(t) s(t-\tau) d t, \\
& =\left\langle s(t) e^{i t \omega}, s(t-\tau)\right\rangle, \\
& =\left\langle s(t) e^{i t \omega}, e^{-i \tau \mathcal{W}} s(t)\right\rangle ;
\end{aligned}
$$

From the Doppler effect perspective, translation is the operation of the frequency operator on the signal, where $\tau$ is the total distance a signal travels to an object, is reflected, and then returns to the receiver.

Using Cohen's notation, we define the time operator, $\mathcal{T}$, as

$$
\mathcal{T}=-\frac{1}{i} \frac{d}{d \omega},
$$

while the frequency operator is

$$
\mathcal{W}=\frac{1}{i} \frac{d}{d t} .
$$

It is understood that these operators act on signals and that

$$
\mathcal{W}^{n} s(t)=\left(\frac{1}{i} \frac{d}{d t}\right)^{n} s(t) .
$$

A very useful calculation trick is based on a modification of Parceval's theorem for an unnormalized signal:

$$
\begin{aligned}
E & =\langle s(t), s(t)\rangle=\frac{1}{2 \pi}\left\langle\left\langle S\left(\omega^{\prime}\right) e^{i \omega^{\prime} t}, 1\right\rangle,\left\langle S(\omega) e^{i \omega t}, 1\right\rangle, 1\right\rangle \\
& =\frac{1}{2 \pi}\left\langle\left\langle\left\langle S\left(\omega^{\prime}\right), 1\right\rangle,\langle S(\omega), 1\rangle\right\rangle e^{i \omega^{\prime} t}, e^{i \omega t}\right\rangle \\
& =\left\langle\left\langle S\left(\omega^{\prime}\right), S(\omega) \delta\left(\omega-\omega^{\prime}\right)\right\rangle\right\rangle=\langle S(\omega), S(\omega)\rangle
\end{aligned}
$$

Now it follows that the expected value of the frequency of a signal $S(\omega)$ can be written as

$$
\begin{aligned}
\langle\omega\rangle & =\int_{-\infty}^{\infty} \omega|S(\omega)|^{2} d \omega \\
& =\frac{1}{2 \pi}\left\langle\omega\left\langle s(t) e^{-i \omega t}, 1\right\rangle,\left\langle s\left(t^{\prime}\right) e^{i \omega t^{\prime}}, 1\right\rangle, 1\right\rangle \\
& =\cdots \\
& =\left\langle s\left(t^{\prime}\right), \mathcal{W} s(t)\right\rangle .
\end{aligned}
$$

From this result, it follows that

$$
\left\langle\omega^{n}\right\rangle=\left\langle s\left(t^{\prime}\right), \mathcal{W}^{n} s(t)\right\rangle,
$$

which can be proved by induction. If $g(t)$ is an analytical function, it follows that

$$
\langle g(\omega)\rangle=\left\langle s\left(t^{\prime}\right), g(\mathcal{W}) s(t)\right\rangle .
$$

Thus, to calculate the average frequency of a function, we do not have to calculate the Fourier transform. Rather one simply calculates derivatives of a function and then integrates. We can thus interpret $e^{i \tau \mathcal{W}}$ as a translation operation on function $s(t)$. A function of time that is translated by amount, $\tau$, can be written as (using the Taylor expansion of function $D=\frac{d}{d t}$ )

$$
s(t+\tau)=e^{\tau D} s(t)=e^{i\left(-i \tau \frac{d}{d t}\right)} s(t)=e^{i \tau \mathcal{W}} s(t)
$$

The frequency translation operator has exactly the same effect:

$$
e^{i \theta \mathcal{T}} S(\omega)=S(\omega+\theta) .
$$

For a complex signal, $s(t)=A(t) e^{i \vartheta(t)}$,

$$
e^{i \tau \mathcal{W}} s(t)=\left(\vartheta^{\prime}(t)-i \frac{A^{\prime}(t)}{A(t)}\right) s(t)
$$

so

$$
\begin{aligned}
\langle\omega\rangle & =\langle S(\omega), \omega S(\omega)\rangle \\
& =\left\langle\left(\vartheta^{\prime}(t)+i \frac{A^{\prime}(t)}{A(t)}\right) A(t), A(t)\right\rangle \\
& =\left\langle\vartheta^{\prime}(t) A(t), A(t)\right\rangle
\end{aligned}
$$

since the second term in the integral is a perfect differential. The average frequency is the derivative of the phase, $\vartheta(t)$, over the density over all time. Thus the phase at each time must be instantaneous in some sense, i.e. $\omega_{i}(t)$, so we can make the identification that $\omega_{i}(t)=\vartheta^{\prime}(t)$. Similarly, we can show that

$$
\begin{aligned}
\left\langle\omega^{2}\right\rangle= & \left\langle S(\omega), \omega^{2} S(\omega)\right\rangle=\left\langle\vartheta^{\prime 2}(t) A(t), A(t)\right\rangle \\
& +\left\langle\left(\frac{A^{\prime}(t)}{A(t)}\right) A(t),\left(\frac{A^{\prime}(t)}{A(t)}\right) A(t)\right\rangle .
\end{aligned}
$$

The covariance of a signal might be thought of as the "average time" multiplied by the instantaneous frequency or

$$
\left\langle t \vartheta^{\prime}(t)\right\rangle=\left\langle t \vartheta^{\prime}(t) A(t), A(t)\right\rangle .
$$

When time and frequency are uncorrelated with each other, then it is reasonable to expect that $\left\langle t \vartheta^{\prime}(t)\right\rangle=\langle t\rangle\langle\omega\rangle$, so the difference between the two is a measure of how time is correlated to the instantaneous frequency. Thus, the covariance of the signal is

$$
\operatorname{Cov}_{t \omega}=\left\langle t \vartheta^{\prime}(t)\right\rangle-\langle t\rangle\langle\omega\rangle,
$$

while the correlation coefficient, $r$, is

$$
r=\frac{\operatorname{Cov}_{t \omega}}{\sigma_{t} \sigma_{\omega}},
$$

which is the normalized covariance. Real signals have zero correlation coefficients as do signals of the form $A(t) e^{i \omega_{0} t}$ 
or $S(\omega)=A(\omega) e^{i \omega t_{0}}$, so signals with complicated phase modulation have a non-zero correlation coefficient.

When dealing with more than one operator acting on a signal, we must be able to interpret the action of multiple operators such as $\mathcal{A B}$ acting upon signals. Here $\mathcal{A B}$ is taken to mean $\mathcal{A}$ acts on the signal followed by $\mathcal{B}$ acting on the signal. When the order of operations does not matter, the operators are said to commute, otherwise the operators don't commute. The commutator of $\mathcal{A}$ and $\mathcal{B}$ is

$$
[\mathcal{A}, \mathcal{B}]=\mathcal{A B}-\mathcal{B A}
$$

while the anti-commutator of $\mathcal{A}$ and $\mathcal{B}$ is

$$
[\mathcal{A}, \mathcal{B}]_{+}=\mathcal{A B}+\mathcal{B A} .
$$

The properties of $[\mathcal{A}, \mathcal{B}]$ are:

1) $[\mathcal{A}, \mathcal{A}]=0$,

2) $[\mathcal{A}, \mathcal{B}]=-[\mathcal{B}, \mathcal{A}]$,

3) $[c \mathcal{A}, \mathcal{B}]=c[\mathcal{A}, \mathcal{B}]$,

4) $[\mathcal{A}, \mathcal{B}+\mathcal{C}]=[\mathcal{A}, \mathcal{B}]+[\mathcal{A}, \mathcal{C}]$

We have adapted the notation of Cohen to define these operators[8]. For example, the action of the time and frequency commutator on a signal is

$$
[\mathcal{T}, \mathcal{W}] s(t)=(\mathcal{T} \mathcal{W}-\mathcal{W} \mathcal{T}) s(t)=[\mathcal{T}, \mathcal{W}] s(t)=i s(t) .
$$

The scale operator $\mathcal{C}$ is defined as

$$
\mathcal{C}=\frac{1}{2}[\mathcal{T}, \mathcal{W}]_{+}=\frac{1}{2}\left(t \frac{d}{d t}+\frac{d}{d t} t\right)
$$

it can also be written as

$$
\mathcal{C}=\mathcal{T} \mathcal{W}+\frac{i}{2}
$$

$\mathcal{C}$ has the property that it transforms a signal $s(t)$ according to

$$
e^{i \sigma \mathcal{C}} s(t)=e^{\sigma / 2} s\left(e^{\sigma / 2} t\right) .
$$

Note that:

1 The effect of this function is to rescale $s(t)$ by the compression factor $e^{\sigma / 2}$.

2 We could also use $\ln \sigma$ in place of $\sigma$ and achieve the same effect: $e^{i \ln \sigma \mathcal{C}} s(t)=\sqrt{\sigma} s(\sqrt{\sigma} t)$.

3 Either form preserves normalization.

4 This operator rescales the amplitude of the broadcast waveform, so it is equivalent to an amplitude reduction of a signal that is measured at a receiver.

From the Doppler effect perspective, translation is the operation of the frequency operator on the signal, where $\tau$ is the total distance a signal travels to an object, is reflected, and then returns to the receiver. The broadband ambiguity function can be rewritten as

$$
\begin{aligned}
& \chi_{W B}(\omega, \tau)=\sqrt{\alpha} \int_{-\infty}^{\infty} s^{*}(t) s(\alpha(t-\tau)) e^{-i t \omega} d t \\
& =\sqrt{\alpha}\left\langle s(t) e^{i t \omega}, s(\alpha(t-\tau))\right\rangle \\
& =\sqrt{\alpha}\left\langle s(t) e^{i t \pi f}, e^{-i \alpha \mathcal{C}} e^{-i \tau \mathcal{W}} s(t) e^{-i t \pi f}\right\rangle,
\end{aligned}
$$

which is the action of operators for scale change $e^{i \ln \alpha \mathcal{C}}$ and the operator for time shift $e^{i \tau \mathcal{W}}$ on the signal. The narrowband ambiguity function can be rewritten as a translation and a scale change acting separately. These 'mathematical operators' change the functional form of the transmitted signal after interaction with the scattering object so the return signal at the receiver can be thought of as a series of operators acting on the transmitted signal. Waveforms that interact with the scattering object can be thought of as the means to measure observables associated with targets. In a sense, we are measuring the "expected value" of an observable when both the transmission and reception portions of the sensor are considered. Recall, the expected value is a concept that occurs in quantum mechanics. In quantum mechanics, the normalized signal is replaced with a function $|\Psi(x)\rangle$ with an associated probability distribution $|\Psi(x)|^{2}=P(x)$. The expected values associated with observable, $\hat{A}$, is calculated as:

$$
\langle\widehat{A}\rangle=\int \widehat{A} P(x) d x=\int \Psi^{*}(x) \widehat{A} \Psi(x) d x=\langle\Psi(x) \widehat{\mid A} \Psi(x)\rangle .
$$

Classical probability has three essential characteristics related to signals:

1) Random Variable $\widehat{x}$ with probability $P(x)$.

2) Expected Value of Observable $\langle\alpha(x)\rangle=\int \alpha(x) P(x) d x$.

3) Change of Variable $\alpha(x)$ given by: $P(\alpha)=\int \delta(\alpha-$ $\alpha(x)) P(x) d x$.

Quantum probability has three essential characteristics related to signals:

1) Probability distribution is $P(\alpha)=|\vartheta(\alpha)|^{2}$

2) Expected Value of Observable $\langle\widehat{A}\rangle=\langle\Psi(x) \widehat{\mid A} \Psi(x)\rangle$.

3) Distribution of one variable from another is

$$
P(\alpha)=|\vartheta(\alpha)|^{2}
$$

where

$$
\vartheta(\alpha)=\int u_{\alpha}^{*}(x) \Psi(x) d x
$$

with $u_{\alpha}(x)$ eigenfunctions of operator $\widehat{A}$ satisfying $\widehat{A} u_{\alpha}(x)=\alpha u_{\alpha}(x)$.

Thus, we define the expected value associated with observable, $\hat{A}$, for a signal $s(t)$ as

$$
\langle\widehat{A}\rangle=\int \widehat{A}|s(t)|^{2} d t=\int s^{*}(t) \widehat{A} s(t) d t=\langle s(t), \widehat{A} s(t)\rangle .
$$

Thus, the wideband ambiguity function can be written as

$$
\chi_{W B}(\omega, \tau)=\sqrt{\alpha}\left\langle e^{-i \alpha \mathcal{C}} e^{-i \tau \mathcal{W}}\right\rangle,
$$

the expected value of the scaling and translation operators for a signal $s(t) e^{-i t \pi f}$, which is equivalent to maximizing the signal to noise ratio (SNR) at the receiver. This way of considering measurement in radar is a natural continuation of the viewpoint that started with Gabour[11], extended by Woodward[1], and Vaidman[10] for considering measurement in radar. 


\section{EXAMPLES}

The reason the ambiguity function is so important in radar waveform analysis is that allows the mathematical engineer to design a radar waveform that has properties which are specific to a desired design criteria or can be used for system tradeoffs for conflicting design criteria. For interactions between the radar signal and the target that extend beyond considerations of position and velocity related criteria, the operator viewpoint may hold some promise. Physical interaction with the target can induce secondary characteristics into the return signal, such as micro-Doppler, which can be incorporated into the design of a receiver to maximize the possibility for detecting these types of secondary target induced characteristics. In additional to a scalar signal, higher dimensional waveforms interactions can be considered as well, such as the polarization of materials affect on the waveform. In addition, we introduce a cross-ambiguity function from the operator perspective which allows the consideration of a new approach to signal amplification.

\section{A. Single Dimensional Signals}

The non-uniform Doppler effect can be used to illustrate this operator viewpoint. The effect of non-uniform Doppler on the radar waveform can be determined by the application of the relativistic boundary conditions to the D'Alembert solution to the wave equation[12]. The scattered waveform in terms of the incident waveform becomes

$$
g(\tau)=-a(h(\tau)) f(2 h(\tau)-\tau)
$$

so $a(t)$ becomes $a\left(h(\tau)=2 h^{\prime}(\tau)-1\right.$. The canonical representation of the scattered waveform

$$
g(\tau)=-\frac{d}{d \tau} F(2 h(\tau)-\tau),
$$

where $F(y)=\int^{y} f(u) d u$.This equation is an exact expression for the scattered waveform independent of any bandwidth assumptions. The effect of the derivative becomes a noticeable effect on wideband waveforms, while it is not on narrowband waveforms. By introducing the two definitions for $\tau$, i.e. $\tau=t+\frac{r(t)}{c}$, and $h(\tau)=t$, as the functional equation $h(\tau)+\frac{r(h(\tau))}{c}=\tau$ which to this function form for the scattered waveform. This change of variable redefines the time axis so "time" starts at $\tau=0$ in the receiver time frame. Solving the functional equation for $h(\tau)$ allows us to accomplish this redefinition.) Micro-Doppler can occur when $h(\tau)$ is a nonlinear function.

For a dynamic system characterized by single parameter $\alpha$, then a dynamic variable $u$ evolves along a path in configuration space. The configuration of the system describes a curve along $\alpha$. Consider the commutator equation

$$
\frac{d u}{d \alpha}=[u, G]
$$

Here, $G$ generates the trajectory $u=u(\alpha)$ and $\alpha$ can be viewed as geometrical parameter. Expanding $u(\alpha)$ in a Taylor series yields [15]

$$
u(\alpha)=u_{0}+\left.\alpha \frac{d u}{d \alpha}\right|_{\alpha=0}+\left.\frac{\alpha^{2}}{2 !} \frac{d^{2} u}{d \alpha^{2}}\right|_{\alpha=0}+\ldots
$$

and using the generator equation to replace the dynamics gives

$$
u(\alpha)=u_{0}+\left.\alpha[u, G]\right|_{\alpha=0}+\left.\frac{\alpha^{2}}{2 !}[[u, G], G]\right|_{\alpha=0}+\ldots .
$$

This can be written as the operator equation[14]

$$
u(\alpha)=\left.\exp (\alpha \hat{G}) u(\alpha)\right|_{\alpha=0},
$$

where

$$
\hat{G} f \doteq[f, G] .
$$

For physical systems, it is evident that the generator of dynamics is time, so any function of time can be thought of as being generated by an operator acting on $u(t)$ :

$$
\begin{aligned}
u(t)= & \left.\exp (t \hat{G}) u(t)\right|_{t=0}=u_{0}+\left.t[u, G]\right|_{t=0} \\
& +\left.\frac{t^{2}}{2 !}[[u, G], G]\right|_{t=0}+\ldots
\end{aligned}
$$

Now any function $u(t)$ can be thought of being generated by an operator $G$. Thus we see how to "generate" any function of a parameter using operator methods[13]. For a given $h(\tau)$, we can assume it is generated by a equation such as $h(\tau)=$ $\left.\exp (\tau \hat{G}) x(\tau)\right|_{\tau=0}$, so

$F(2 h(\tau)-\tau)=F\left(\left.2 \exp (\tau \hat{G}) x(\tau)\right|_{\tau=0}-\tau\right)=\exp (\tau \widehat{H}) s(\tau)$,

where $\widehat{H}$ depends on the specifics of the interaction.

For example, $\widehat{H}$ would be a comb operator in the frequency domain for a periodic function. In this case, we are estimating the expected value of $\langle\exp (\tau \widehat{H})\rangle$ at the receiver. Since any scalar interaction on the waveform can be thought of as the action of an operator on the broadcast waveform, a more general ambiguity function can always be defined as

$$
\chi_{O}(\omega, \tau)=\frac{A}{\sqrt{\pi}} \int_{-\infty}^{\infty} e^{-i t \omega} s^{*}(t) \exp (\tau \widehat{H}) s(t) d t
$$

or

$$
\chi_{O}(\omega, \tau)=\left\langle e^{-i t \omega} s^{*}(t), \exp (\tau \widehat{H}) s(t)\right\rangle .
$$

The key to understanding measurement of more complicated interactions is combining the kernel

$$
\left\langle s(t) e^{i t \omega}, e^{i \ln \sigma \mathcal{C}} e^{i \tau \mathcal{W}} s(t)\right\rangle .
$$

with additional signal interactions interpreted as the action of additional operators in this kernel:

$\left\langle s(t) e^{i t \omega}, \exp \left(\tau \widehat{H}_{1}\right) \exp \left(\tau \widehat{H}_{2}\right) \ldots \exp \left(\tau \widehat{H}_{n}\right) e^{i \sigma \mathcal{C}} e^{i \tau \mathcal{W}} s(t)\right\rangle$,

thus for multiple interactions, the multiple interaction ambiguity function is

$$
\begin{aligned}
& \chi_{O_{1, . . n}}(\omega, \tau) \\
= & \left\langle s(t) e^{i t \omega}, \exp \left(\tau \widehat{H}_{1}\right) \exp \left(\tau \widehat{H}_{2}\right) \ldots \exp \left(\tau \widehat{H}_{n}\right) e^{i \sigma \mathcal{C}} e^{i \tau \mathcal{W}} s(t)\right\rangle .
\end{aligned}
$$


This approach may be the key to determining underlying interactions that can be deeply buried within the return signal.

\section{B. Cross Operator Ambiguity Functions}

The cross ambiguity function (CFA) in its' symmetric form is defined as

$$
\chi_{r, g}(\omega, \tau)=\int_{-\infty}^{\infty} e^{-i t \omega} r^{*}\left(t+\frac{\tau}{2}\right) s\left(t-\frac{\tau}{2}\right) d t,
$$

where $s(t)$ is the transmitted signal, while is the correlation signal $r(t)$. This is the traditional form for the CFA. Instead of this form, a new type of CFA is proposed based on quantum mechanics. Any signal can be expressed as a complex vector. A new approach to signal amplification based on work by Aharonov on amplification of the measurement of some operators in quantum phenomena [16]. Since any quantity that involves the usage of expected values of complex signals can be expressed in the same mathematical form as the quantum mechanical approach to signal amplification, the Aharonov approach suggests a potential candidate for the signal amplification that is similar to a CFA. The classical equivalent to this is what we choose to call cross correlation signal amplification. The definition of the cross correlation amplification of an observable $\widehat{A}$ of a waveforms $\left|\Psi_{i}\right\rangle$ and $\left|\Psi_{i}\right\rangle$ are:

$$
{ }_{f}\left\langle\mathcal{A}_{\text {cross }}\right\rangle_{i}=\frac{\left\langle\Psi_{f}|\widehat{A}| \Psi_{i}\right\rangle}{\left\langle\Psi_{f} \mid \Psi_{i}\right\rangle}
$$

One can think of this is that we are measuring observable with $\widehat{A}$ waveform $\left|\Psi_{i}\right\rangle$ while we correlate the return signal $\widehat{A}\left|\Psi_{i}\right\rangle$ with correlation waveform $\left|\Psi_{f}\right\rangle$. As an example of amplification, define two operators: the signal splitting operators

$$
\mathcal{W}_{+}=\left[\begin{array}{ll}
1 & 0 \\
0 & \epsilon
\end{array}\right], \text { and } \mathcal{W}_{-}=\left[\begin{array}{cc}
\epsilon & 0 \\
0 & 1
\end{array}\right] \text {, }
$$

while the component selection operators are defined as

$$
\mathcal{S}_{+}=\left[\begin{array}{ll}
1 & 0 \\
0 & 0
\end{array}\right], \quad \text { and } \mathcal{S}_{-}=\left[\begin{array}{ll}
0 & 0 \\
0 & 1
\end{array}\right] \text {. }
$$

The input signals can be defined in two different ways, as

$$
\left|\Psi^{P}(t)\right\rangle_{\text {in }}^{+}=\mathcal{W}_{+}|\Psi(t)\rangle_{P} \quad \text { or } \quad\left|\Psi^{P}(t)\right\rangle_{\text {in }}^{-}=\mathcal{W}_{-}|\Psi(t)\rangle_{P},
$$

or

$$
\left|\Psi^{I Q}(t)\right\rangle_{\text {in }}^{+}=\mathcal{W}_{+}|\Psi(t)\rangle_{P} \quad \text { or } \quad\left|\Psi^{I Q}(t)\right\rangle_{\text {in }}^{-}=\mathcal{W}_{-}|\Psi(t)\rangle_{P}
$$

The output signals are defined as

(Form B)

$$
\left|\Psi^{P}(t)\right\rangle_{\text {out }}^{+}=\mathcal{S}_{+}|\Psi(t)\rangle_{P} \quad \text { or } \quad\left|\Psi^{P}(t)\right\rangle_{\text {out }}^{-}=\mathcal{S}_{-}|\Psi(t)\rangle_{P}
$$

or

(Form A')

$$
\left|\Psi^{I Q}(t)\right\rangle_{\text {out }}^{+}=\mathcal{S}_{+}|\Psi(t)\rangle_{P} \quad \text { or } \quad\left|\Psi^{I Q}(t)\right\rangle_{\text {out }}^{-}=\mathcal{S}_{-}|\Psi(t)\rangle_{P} .
$$

(Form B')

Now on a purely theoretical level, if we assume that one of the four possible $|\Psi(t)\rangle_{\text {in }}$ are used to make a measurement
$\mathcal{M}$ of some property of a distant object, the effect of the measurement can be signal amplification.

We can define the cross correlation measurement amplification of measurement operator as

$$
{ }_{f}\left\langle\mathcal{M}_{\text {cross }}\right\rangle_{i}=\frac{\text { out }\langle\Psi(t)|\mathcal{M}| \Psi(t)\rangle_{\text {in }}}{\text { out }\langle\Psi(t) \mid \Psi(t)\rangle_{\text {in }}} .
$$

There are 16 possible versions of weak, but one is sufficient to illustrate the point. One achieves $\epsilon$-phasor amplification of $\mathcal{M}$ by the choice

$$
{ }_{f}\left\langle\mathcal{M}_{\text {cross }}\right\rangle_{i}=\frac{-{ }_{\text {out }}\left\langle\Psi^{P}(t)|\mathcal{M}| \Psi^{P}(t)\right\rangle_{\text {in }}^{+}}{{ }_{\text {out }}\left\langle\Psi^{P}(t) \mid \Psi^{P}(t)\right\rangle_{\text {in }}^{+}} .
$$

Carrying out the computation gives

$$
\begin{aligned}
& { }_{f}\left\langle\mathcal{M}_{\text {cross }}\right\rangle_{i}=\frac{-\underset{\text { out }}{ }\left\langle\Psi^{P}(t)|\mathcal{M}| \Psi^{P}(t)\right\rangle_{\text {in }}^{+}}{\left[\begin{array}{ll}
x(t) & y(t)
\end{array}\right]\left[\begin{array}{c}
0 \\
\epsilon y(t)
\end{array}\right]} \\
& =\frac{{ }_{\text {out }}\left\langle\Psi^{P}(t)|\mathcal{M}| \Psi^{P}(t)\right\rangle_{\text {in }}^{+}}{\epsilon y^{2}(t)} \text {. }
\end{aligned}
$$

Thus, one gets amplification of ${ }_{\text {out }}^{-}\left\langle\Psi^{P}(t)|\mathcal{M}| \Psi^{P}(t)\right\rangle_{\text {in }}^{+}$provided $\epsilon y^{2}(t)<1$. This will not always be true so the conditions under which it is true need to be investigated. One achieves $\epsilon-\mathrm{I} / \mathrm{Q}$ amplification of $\mathcal{M}$ by the choice

$$
\begin{aligned}
& { }_{f}\left\langle\mathcal{M}_{\text {cross }}\right\rangle_{i}=\frac{{ }_{\text {out }}\left\langle\Psi^{I Q}(t)|\mathcal{M}| \Psi^{I Q}(t)\right\rangle_{\text {in }}^{+}}{{ }_{\text {out }}\left\langle\Psi^{I Q}(t) \mid \Psi^{I Q}(t)\right\rangle_{\text {in }}^{+}} \\
& =\frac{\overline{\text { out }}\left\langle\Psi^{I Q}(t)|\mathcal{M}| \Psi^{I Q}(t)\right\rangle_{\text {in }}^{+}}{\epsilon Q^{2}(t)} .
\end{aligned}
$$

Thus, one gets amplification of ${ }_{\text {out }}^{-}\left\langle\Psi^{I Q}(t)|\mathcal{M}| \Psi^{I Q}(t)\right\rangle_{\text {in }}^{+}$ provided $\epsilon Q^{2}(t)<1$.

Another example to illustrate this is polarization. Polarization can only be measured with special radars, such as certain types of SAR radars, none the less, the expectation value is equivalent to that of taking the expected values of a spin matrix in quantum mechanics. Shape and size are multi-dimensional attributes of scattering objects that have multi-dimensional versions of the delay operators. There is another aspect of the operator formalism to discuss related to physics. Any nonscalar return signal has components that can be written like this. Consider a broadcast waveform of the form

$$
\left|\Psi_{i}\right\rangle=\frac{1}{\sqrt{2}}\left[\begin{array}{c}
\exp (i \alpha / 2) \\
\exp (-i \alpha / 2)
\end{array}\right]
$$

while the cross correlation waveform is

$$
\left|\Psi_{f}\right\rangle=\frac{1}{\sqrt{2}}\left[\begin{array}{c}
\exp (-i \alpha / 2) \\
\exp (i \alpha / 2)
\end{array}\right]
$$

The operator we wish to observe is a polarization operator, which can be represented by the matrix

$$
\widehat{A}=\sigma_{1}=\left[\begin{array}{ll}
0 & 1 \\
1 & 0
\end{array}\right]
$$




$$
\begin{aligned}
\langle\widehat{A}\rangle_{\text {weak }} & =\frac{\left\langle\Psi_{f}|\widehat{A}| \Psi_{i}\right\rangle}{\left\langle\Psi_{f} \mid \Psi_{i}\right\rangle} \\
& =\frac{\frac{1}{2}\left[\begin{array}{c}
\exp (i \alpha / 2) \\
\exp (-i \alpha / 2)
\end{array}\right]^{T}\left[\begin{array}{c}
\exp (-i \alpha / 2) \\
\exp (i \alpha / 2)
\end{array}\right]}{\frac{\exp (i \alpha)+\exp (-i \alpha)}{2}} \\
& =\frac{1}{\cos \alpha}
\end{aligned}
$$

which magnifies one particular polarization component by an arbitrary amount. This example of amplification, which is analogous to spin systems in quantum mechanics, can be found polarimetric radars. The general form of a polarization matrix can be written as the sum of the four polarization matrices

$$
\begin{gathered}
\sigma_{0}=\left[\begin{array}{ll}
1 & 0 \\
0 & 1
\end{array}\right], \quad \sigma_{1}=\left[\begin{array}{cc}
1 & 0 \\
0 & -1
\end{array}\right], \\
\sigma_{2}=\left[\begin{array}{ll}
0 & 1 \\
1 & 0
\end{array}\right], \quad \sigma_{3}=\left[\begin{array}{cc}
0 & -i \\
i & 0
\end{array}\right] .
\end{gathered}
$$

Amplification works for any of these operators as well. The polarization matrix $\Psi$ for an electromagnetic interaction can be written as the sum of three matrices:

$$
\Psi=\frac{1}{2} \sum_{j}^{3} S_{j} \sigma_{j}
$$

where $S_{0}=I, S_{1}=I p \cos 2 \psi \cos 2 \chi, S_{2}=I p \sin 2 \psi \cos 2 \chi$, $S_{3}=I p \sin 2 \chi$. Moderate power radars, which have a variety of applications to the remote sensing and for search and rescue applications, can be written as a superposition of these type of operators, so the ability to amply would help detect hidden objects in background.

This suggest that one could define an Aharonov cross ambiguity function for an operator $\widehat{A}$ as

$$
\chi_{r, s}^{A}(\omega, \tau)=\frac{\left\langle e^{-i t \omega} r^{*}(t), \exp (\tau \widehat{A}) s(t)\right\rangle}{\left\langle e^{-i t \omega} r^{*}(t), s(t)\right\rangle},
$$

which can be readily generalized to vector signals. Note this type of cross ambiguity function would lead to new ways to design waveforms which are significantly different than might be considered by traditional approaches to waveform design. This new approach will be considered in a future paper.

\section{CONCLusions}

The viewpoint that an ambiguity function can be interpreted as the expected value of an operator widens the horizon for thinking about the waveform target interaction. The operator method is a much richer way to look at the radar measurement problem because of its ability to produce a wide variety of distributions associated with the information contained in a signal. In particular, it is possible to put the ambiguity function in a wider context as part of a general theory of measurement. There is a much greater freedom of description of the same physical situation which suggests that we can find information present in waveforms that a waveform designer would not think to look for. This approach to incorporating quantum mechanical ideas has been championed by Baraniuk[17],[18] recently by extending the Hermitian operator approach in quantum mechanics to unitary operators in signal processing. The specifics of the type of operators matter relative to the physics of the interaction of the target with the waveform, so this may be important for future extensions of this work.

Acknowledgement 1: Thanks to Greg Coxson for encouraging the writing of this paper. The concepts discussed in this paper arose after hearing a talk by Y. Aharonov and latter discussions with him about the concepts of weak amplification of classical signals.

\section{REFERENCES}

[1] P. M. Woodward, Probability and Information Theory with Applications to Radar, Artech House Publishers, 1980.

[2] D. O. North, "An analysis of the factors which determine signal/noise discrimination in pulsed carrier systems". RCA Labs., Princeton, NJ, Rep. PTR-6C, 1943.

[3] A. Papoulis, Signal Analysis, McGraw-Hill, 1977.

[4] A. Papoulis, S. U. Pillai, Probability, Random Variables, and Stochastic Processes, $4^{\text {th }}$ Ed. McGraw-Hill, New York, 2002.

[5] H. L. Van Trees, Detection, Estimation, and Modulation Theory, Part I, Wiley-Interscience; Reprint edition, 2002.

[6] A. Papoulis, The Fourier Integral and its Applications, McGraw-Hill, 1961.

[7] N. Levanon and E. Mozeson, Radar Signals, Wiley-IEEE Press, 2004.

[8] L. Cohen, Time-Frequency Analysis, Prentice-Hall, 1995.

[9] L. Cohen, The Scale Representation, IEEE Transactions on Signal Processing, Vol. 41, NO. 12. December., 1993

[10] D. E. Vakman, Sophisticated Signals and the Uncertainty Principle in Radar, Springer-Verlag New York Inc., 1968.

[11] D. Gabor, "Communication theory and physics", Information Theory, Transactions of the IRE Professional Group on Information, Feb. 1953, Volume: 1, Issue: 1.

[12] J. E. Gray and S. R. Addison, "Effect of Non-uniform Motion on the Doppler Spectrum of Scattered Continuous Waveforms," IEE Proceedings-Radar, Sonar and Navigation, August 2003, Vol.150, Issue 4.

[13] F. M Fernandez, Operator Methods in Classical Mechanics, Am. J. Phys., 70 (9), September, 2002.

[14] T. F. Jordan, "Steppingstones in Hamiltonian Dynamics", Am. J. Phys., 72 (8), August 2004.

[15] C. F. de Souza and M. M. Gandelman, An Algebraic Approach for Solving Mechanical Problems, Am. J. Phys., 58 (5), May 1990.

[16] Y. Aharonov and D. Rohrlich, Quantum Paradoxes: Quantum Theory for the Perplexed, Wiley-VCH, 2005.

[17] R. G. Baraniuk, Unitary Equivalence: A New Twist on Signal Processing, IEEE TRANSACTIONS ON SIGNAL PROCESSING, VOL. 43, NO. 10, OCTOBER 1995.

[18] R. G. Baraniuk, "Beyond Time-Frequency Analysis: Energy Densities in One and Many Dimensions", IEEE TRANSACTIONS ON SIGNAL PROCESSING, VOL. 46, NO. 9, SEPTEMBER 1998 